\title{
Guía de Práctica Clínica: Recomendaciones para el rastreo del glaucoma primario de ángulo abierto
}

\author{
Recommendations for screening of primary open-angle glaucoma
}

U.S. Preventive Services Task Force; Screening for Glaucoma. Annals of Internal Medicine. 2013 Oct;159 (7):484-489

\section{Desarrollo de la guía}

Las recomendaciones de esta guía están dirigidas a adultos sin síntomas visuales que se atienden en el ámbito de la atención primaria.

El grupo revisor no encontró evidencia concluyente respecto de la precisión del rastreo de glaucoma primario de ángulo abierto en adultos. Esta ausencia se debe a que no existe un estándar de oro (gold standard) diagnóstico definido contra el cual pueda compararse la precisión de estos métodos de cribado.

Si bien se sabe que cualquier estrategia de rastreo de asocia a resultados falsos positivos y negativos, en este caso no se conoce su magnitud dada la enorme variabilidad reportada de sus características operativas (especificidad y sensibilidad). Por ejemplo, la tonometría (medición de la presión intraocular), que es el método de rastreo más utilizado, es inadecuada por sí sola, ya que algunos individuos con presión intraocular normal pueden desarrollar glaucoma, mientras que algunos con aumento de la misma no lo desarrollarán nunca. Otros métodos de evaluación utilizados por los especialistas (evaluación de campos visuales y exámenes de la papila óptica) reportan variabilidad en la precisión y la confiabilidad para el diagnóstico y la detección de la progresión de la enfermedad.

Vale destacar que una estrategia de rastreo poblacional inade- cuada puede conducir a enormes cantidades de pacientes innecesariamente tratados y a un aumento de la incidencia de efectos adversos.

Si bien no se encontraron estudios que hayan considerado los daños del rastreo, numerosos trabajos evaluaron los efectos adversos del tratamiento médico (enrojecimiento, ardor, irritación, sequedad ocular, aumento de la pigmentación del iris y edema macular cistoideo) y del tratamiento quirúrgico (hipotonía, aplanamiento de la cámara anterior, catarata, desprendimiento coroideo, infección y sangrado).

Si bien existen trabajos que respaldan que el tratamiento precoz de la presión intraocular elevada y del glaucoma temprano disminuyen la incidencia de defectos en el campo visual y/o el empeoramiento de defectos ya presentes; el grupo revisor no encontró evidencia directa de que globalmente el rastreo implique beneficios en la calidad de vida de las personas y/o en alteraciones objetivas de la visión.

Esta evidencia de beneficio debería provenir de algún ensayo clínico aleatorizado y controlado que evalúe contra el cuidado estándar la potencial estrategia de rastreo de glaucoma (o de sus factores de riesgo) a implementar. Ver tabla 1.

Tabla 1. resumen de la recomendación de la Fuerza de Tareas de Servicio de Preventivos de EE.UU. para el rastreo del glaucoma.

\begin{tabular}{l|l}
\hline Población & Adultos sin síntomas visuales atendidos en atención primaria. \\
\hline Recomendación & $\begin{array}{l}\text { No hay recomendación. La USPTF considera que falta evidencia o es de baja calidad, o es conflictiva, y el equilibrio entre los beneficios y } \\
\text { los daños no puede determinarse. Si se ofrece el servicio, los pacientes deben comprender dicha incertidumbre. }\end{array}$ \\
\hline Evaluación del riesgo & $\begin{array}{l}\text { Factores de riesgo importantes para el glaucoma primario de ángulo abierto: presión intraocular elevada, edad avanzada, antecedentes } \\
\text { familiares de glaucoma, raza afro-americana. }\end{array}$ \\
\hline Estudios de rastreo & $\begin{array}{l}\text { El diagnóstico del glaucoma se realiza en base a varios estudios que, al combinarse, evalúan la estructura biológica y la función del } \\
\text { nervio óptico y la presión intraocular (PI0). La mayoría de los estudios disponibles en un ámbito de atención primaria no tienen una } \\
\text { precisión aceptable como para detectar glaucoma. }\end{array}$ \\
\hline Tratamiento & $\begin{array}{l}\text { El objetivo fisiológico inicial y medición del efecto del tratamiento primario del glaucoma es la reducción de la PI0. Los tratamientos } \\
\text { efectivos incluyen medicamentos, láser y cirugía. Sin embargo, implican daños potenciales, y su efectividad para reducir los } \\
\text { impedimentos de la función visual percibidos por el paciente son inciertos. }\end{array}$ \\
\hline Equilibrio entre beneficios y daños & $\begin{array}{l}\text { La evidencia respecto de la precisión de los estudios de rastreo, especialmente en el ámbito de la atención primaria, y los beneficios del } \\
\text { rastreo para retrasar o impedir el trastorno visual o mejorar la calidad de vida es insuficiente. Por lo tanto, la certeza global de la } \\
\text { evidencia es baja y la USPTF no puede determinar el equilibrio entre beneficios y daños del rastreo del glaucoma en adultos } \\
\text { asintomáticos. }\end{array}$ \\
\hline
\end{tabular}

PIO: presión intraocular.

\section{Recomendación final}

La USPSTF concluye que dada la incertidumbre de la magnitud de los beneficios del tratamiento temprano y los conocidos potenciales daños del rastreo y del tratamiento temprano, no se puede determinar el equilibrio entre los beneficios y los daños del rastreo de glaucoma primario de ángulo abierto en adultos.
Fuente de financiamiento: La USPSTF es un organismo independiente y voluntario. El Congreso de los Estados Unidos obliga a que la Agencia de Investigación y Calidad en Salud financie las operaciones de la USPSTF.

Conflictos de interés potenciales: Ninguno declarado. Los formularios de declaración de miembros de la USPSTF pueden verse en:

www.acponline.org/authors/icmje/ConflictOfInterestForms.do? msNum=M13-1387. 


\section{Comentario}

El glaucoma primario de ángulo abierto es una enfermedad ocular crónica, progresiva y generalmente bilateral, que comienza durante la adultez, afecta el ángulo de la cámara anterior y se presenta con evidencia de daño del nervio óptico (alteraciones estructurales de la papila óptica o de la capa de fibras nerviosas retinianas y/o alteraciones confiables y reproducibles del campo visual).

Para establecer su diagnóstico es importante que no haya otra explicación alternativa de los cambios "glaucomatosos" del nervio óptico ${ }^{1}$.

Una revisión de estudios de prevalencia estimó que, actualmente, este tipo de glaucoma afecta a 45 millones de personas en el mundo y es la segunda causa mundial de ceguera, lo que implica que haya 4,5 millones de personas ciegas por glauco$\mathrm{ma}^{2}$. Dado que el glaucoma no provoca síntomas en sus estadios iniciales y cuando éstos se producen, generalmente ya se ha dañado significativamente el nervio óptico; y que su tratamiento en etapas tempranas retrasa el daño al nervio óptico y la pérdida de la visión (y/o del campo visual ${ }^{3-5}$ ); es necesario consensuar una estrategia de rastreo que permita identificar a los pacientes en etapas iniciales de la enfermedad o en riesgo de sufrir glaucoma para reducir el impacto de esta patología.

El glaucoma representa una de las principales causas de ceguera y para disminuir el impacto de esta enfermedad es necesaria su detección precoz.

Sin embargo, el diagnóstico precoz enfrenta varias dificultades que se deben a múltiples factores: 1) el glaucoma es asintomático hasta estadios avanzados de la enfermedad; 2) los métodos de evaluación del daño y la progresión tienen confiabilidad variable; 3 ) algunos pacientes con presión intraocular normal desarrollan daño glaucomatoso, por lo que una medición de presión ocular dentro de límites normales no excluye el diagnóstico de enfermedad.

A pesar de que se considera que la causa del daño glaucomatoso es multifactorial, su causa exacta no se conoce hasta la actualidad.

El único factor asociado a daño glaucomatoso tratable para retrasar la progresión del daño es la presión intraocular ${ }^{1}$, y varios estudios apoyan la indicación de reducir la presión intraocular en pacientes con glaucoma: El Estudio sobre la Intervención en el Glaucoma Avanzado fue uno de los primeros ensayos clínicos (incluyó 789 ojos, con ocho a 11 años de seguimiento) y documentó que a menor presión intraocular, menor es el riesgo de progresión del campo visual ${ }^{6}$.

El Estudio sobre el Tratamiento de la Hipertensión Ocular fue el primero en demostrar que el tratamiento de la presión intraocular retrasa o previene el comienzo del daño glaucomatoso. Fue un ensayo clínico aleatorizado en el cual participaron 1636 pacientes con presiones intraoculares entre 24 y 32 $\mathrm{mmHg}$ y comparó el efecto del tratamiento de la PIO contra la observación sin tratamiento. Los pacientes fueron seguidos durante diez años ${ }^{7}$.

El Ensayo sobre el Glaucoma Manifiesto Temprano, que comparó el tratamiento del glaucoma (reduciendo la presión intraocular) versus la observación sin tratamiento hasta la aparición de signos de progresión en 255 pacientes con glaucoma temprano, con un seguimiento de siete a 11 años, comunicó que la progresión era significativamente más lenta en los pacientes tratados ${ }^{8}$.

El Estudio Colaborativo del Glaucoma de Tensión Normal, que comparó el tratamiento para reducción de la presión intraocular versus el no tratamiento en 140 pacientes con glaucoma de tensión normal (esto es daño glaucomatoso con presión intraocular menor de $24 \mathrm{mmHg}$ ), con un seguimiento de más de cinco años, obtuvo resultados similares ${ }^{9}$. Por lo tanto, sería ideal contar con un método de rastreo preciso, sencillo y económico, que permitiera detectar pacientes con glaucoma en los estadios más precoces posible.

Los tres abordajes existentes para el rastreo del glaucoma: medición de la presión intraocular, evaluación de la papila óptica y la capa de fibras nerviosas retinianas, y evaluación del campo visual, proveen datos complementarios, pero realizar los tres en una única consulta es impráctico dado el tiempo total que requiere realizarlos.

Además, son costosos y difíciles de realizar (por la tecnología y la experiencia que requiere su realización e interpretación) para un ámbito de atención primaria.

Por otro lado, la realización de cada uno por separado aportaría datos incompletos para la detección de la enfermedad o de su progresión. La medición de la presión intraocular, uno de los métodos más utilizados por su bajo costo y simplicidad, es inefectiva para la detección del glaucoma en campañas de rastreo poblacionales ${ }^{1}$

Por ejemplo, utilizando el valor de corte habitual de $22 \mathrm{mmHg}$, estudios poblacionales han demostrado que un significativo número de pacientes con diagnóstico de glaucoma cursan con presiones intraoculares menores a este valor, en una medición única o en mediciones sucesivas ${ }^{10-11}$.

El rastreo sería más útil y costo-efectivo si se dirigiera a los grupos de población que presentan factores de riesgo para desarrollar neuropatía óptica glaucomatosa.

Estos son: 1) edad avanzada, 2) antecedentes familiares de glaucoma, 3) ascendencia africana o etnicidad latina/hispánica, 4) hipertensión arterial, 5) diabetes tipo 2, 6) miopía alta.

El rastreo de estos pacientes de mayor riesgo debería incluir un examen completo realizado por un oftalmólogo ${ }^{12}$, con especial atención a aquellos factores que definen el diagnóstico, el curso y el tratamiento de la enfermedad ${ }^{13}$ : historia clínica, agudeza visual, examen de las pupilas y del segmento anterior del ojo, medición de la presión intraocular, gonioscopía (evaluación del ángulo de la cámara anterior) y fondo de ojo con evaluación de las papilas ópticas y de la capa de fibras nerviosas retinianas.

Estos estudios deberán complementarse, de acuerdo a la evaluación del oftalmólogo, con mediciones del espesor corneal central, campos visuales y análisis de la papila óptica y las capa de fibras nerviosas retinianas. En este grupo de riesgo, la Academia Americana de Oftalmología recomienda realizar la evaluación cada uno a tres años en personas de 40 a 54 años de edad, cada uno a dos años entre los 55 y 64 años de edad, y cada seis a 12 meses en los mayores de 65 años. Además, provee recomendaciones para el control oftalmológico del paciente asintomático y sin factores de riesgo conocidos ${ }^{12}$.

\section{Conclusiones de la comentadora}

La evidencia actual sobre el rastreo del glaucoma no avala una recomendación de rastreo masivo en ámbitos de atención primaria. El interrogatorio por parte del médico de atención primaria sobre factores de riesgo y la recomendación dirigida al paciente (aún asintomático, con factores de riesgo conocidos o sin ellos) de una evaluación completa periódica por el oftalmólogo permitiría un diagnóstico más precoz y un tratamiento oportuno del glaucoma. 
Ana Gabriela Palis [ Servicio de Oftalmología, Hospital Italiano de Buenos Aires, Argentina. Gabriela.palis @ hospitalitaliano.org.ar]

Palis A. Recomendaciones para el rastreo del glaucoma primario de ángulo abierto. Evid Act Pract Ambul. Ene-Mar 2014;17(1)34-36. Comentado de: Moyer V and U.S. Preventive Services Task Force; Screening for Glaucoma: U.S. Preventive Services Task Force Recommendation Statement. Annals of Internal Medicine. 2013 Oct;159(7):484-489. PMID: 23836133.

\section{Referencias}

1. American Academy of Ophthalmology Glaucoma Panel. Preferred Practice Pattern Guidelines. Primary Open-Angle Glaucoma. San Francisco, CA: American Academy of Ophthalmology; 2010. Disponible en: www.aao.org/ppp.

2. Quigley Hy col. The number of people with glaucoma worldwide in 2010 and 2020. Br J Ophthalmol 2006; 90:262-7.

3. Boland M y col. Treatment for Glaucoma: Comparative Effectiveness. Comparative Effectiveness Review no. 60. ARHW publication no. 12-EHC038-EF. Rockville, MD: Agency for Healthcare Research and Quality; April 2012.

4. Vass $C$ y col. Medical interventions for primary open angle glaucoma and ocular hypertension. Cochrane Database Syst Rev. 2007:CD003167 [PMID: 17943780]

5. Maier Py col. Treatment of ocular hypertension and open angle glaucoma: meta-analysis of randomised controlled trials. BMJ 2005; 331:134. [PMID: 15994659]

6. The AGIS Investigators. The Advanced Glaucoma Intervention Study (AGIS): 7. The relationship between intraocular pressure and visual field deterioration. Am J Ophthalmol 2000; 130:429-40.

7. Kass M y col. The Ocular Hypertension Treatment Study: a randomized trial determines that topical ocular hypotensive medication delays or prevents the onset of primary open angle glaucoma. Arch Ophthalmol 2002; 120:701-13.

8. Heijl A y col. Early Manifest Glaucoma Trial Group. Arch Ophthal 2002; 120:1268-79.

9. Collaborative Normal-Tension Glaucoma Study Group. Comparison of glaucomatous progession between untreated patients with nomal-tension glaucoma and patients with therapeutically reduced intraocular pressures. Am J Ophthalmol 1998; 126: 487-97.

10. Dielemans I y col. The prevalence of open-angle glaucoma in a population-based study in The Netherlands. The Rotterdam Study. Ophthalmology 1994; 101:1851-5.

11. Mitchell P y col. Prevalence of open-angle glaucoma in Australia. The Blue Mountains Eye Study. Ophthalmology 1996; 103:1661-9.

12. American Academy of Ophthalmology Preferred Practice Pattemes Committee. Preferred Practice Pattem® Guidelines. Comprehensive Adult Medical Eye Evaluation. San Francisco, CA: American Academy of Ophthalmology; 2010. Disponible en: www.aao.org/ppp.

13. American Academy of Ophthalmology Glaucoma Panel. Preferred Practice Pattern Guidelines. Primary Open-Angle Glaucoma Suspect. San Francisco, CA: American Academy of Ophthalmology; 2010. Disponible en: www.aao.org/ppp. 